\title{
A Prospective Cohort Study of Perceived Noise Exposure at Work and Cerebrovascular Diseases among Male Workers in Japan
}

\author{
Yoshihisa Fujino ${ }^{1}$, Hiroyasu $\mathrm{IsO}^{2}$, Akiko TAmakoshi ${ }^{3}$, for the JACC study group ${ }^{4}$
}

${ }^{1}$ Department of Preventive Medicine and Community Health, University of Occupational and Environmental Health, Japan, ${ }^{2}$ Public Health, Department of Social and Environmental Medicine, Graduate school of Medicine, Osaka University, ${ }^{3}$ Department of Epidemiology, National Institute for Longevity Sciences, ${ }^{4}$ See acknowledgments for the investigators (name and affiliation) involved in the JACC Study, Japan

\begin{abstract}
A Prospective Cohort Study of Perceived Noise Exposure at Work and Cerebrovascular Diseases among Male Workers in Japan: Yoshihisa FuJINo, et al. Department of Preventive Medicine and Community Health, University of Occupational and Environmental Health, Japan-This study prospectively examined the association between perceived noise exposure at work and cerebrovascular diseases among Japanese male workers. A baseline survey was conducted between 1988 and 1990, which involved 110,792 inhabitants (age range: $40-79 \mathrm{yr}$ ) from 45 areas throughout Japan. Subsequent causes of death were identified from death certificates. The analysis was restricted to 14,568 men free of a cerebrovascular diseases (age range: 40-59 yr) who were in work at the time of the baseline survey. All subjects completed a self-administered questionnaire at the baseline. This included a question regarding perceived noise exposure at work. The Cox proportional-hazards model was used to estimate the risks of perceived noise exposure for death due to cerebrovascular diseases. The model included age, smoking, alcohol consumption, educational level, perceived mental stress, past medical history, body mass index, hours of walking, hours of exercise, shift work, and job type. During the 190,777 person-years of follow-up, a total of 1,064 deaths were recorded, 98 from cerebrovascular diseases, 27 deaths from subarachnoid haemorrhage, 35 deaths from intracerebral haemorrhage, and 25 deaths from cerebral infarction. Noise exposure did not increase the risk of cerebrovascular diseases, subarachnoid haemorrhage, or cerebral infarction. However, perceived noise exposure increased the risk of
\end{abstract}

Received Jan 12, 2007; Accepted Jun 28, 2007

Correspondence to: Y. Fujino, Department of Preventive Medicine and Community Health, University of Occupational and Environmental Health, Japan, 1-1 Iseigaoka, Yahatanishiku, Kitakyushu 807-8555, Japan (e-mail: zenq@med.uoeh-u.ac.jp) intracerebral haemorrhage diseases (hazard ratio $(\mathrm{HR})=2.38,95 \% \mathrm{Cl}: 1.20,4.71, p=0.013)$. Furthermore, individuals with hypertension were highly susceptible to the effect of perceived noise exposure on the risk of intracerebral hemorrhage, but this association was not observed among the subjects without hypertension. Although the underlying mechanisms are not clear, hypertensive individuals with perceived noise exposure at work should be regarded as a high-risk group for intracerebral hemorrhage.

(J Occup Health 2007; 49: 382-388)

Key words: Cerebrovascular disorders, Cohort studies, Japan, Noise, Stress

Noise exposure is one of the major occupational hazards in many places, and has several health effects, including hearing loss and psychological effects such as sleep disturbances, annoyance, and mental stress. In addition, previous studies have suggested that noise exposure is associated with blood pressure changes and cardiovascular diseases ${ }^{1-6)}$. A recent meta-analysis showed that occupational noise exposure was significantly associated with hypertension and cardiovascular diseases ${ }^{6}$. Although the biological mechanism of this association is complex, noise-induced cardiovascular diseases and increased blood change are considered a consequence of stress ${ }^{6}$. Stress may lead to increased ambulatory blood pressure levels and pulse rates $^{7)}$, reduced insulin sensitivity ${ }^{8}$, increased platelet aggregation $^{9)}$, and endothelial dysfunction ${ }^{10)}$, as a result of activating the sympathetic nerve system.

It is well known that both cardiovascular diseases and cerebrovascular diseases have similar risk profiles, including lifestyle factors and socioeconomic factors. Particularly, stress is considered an important risk factor for cerebrovascular diseases as well as coronary heart disease $^{11-17)}$. We therefore hypothesized that noise 
exposure may increase the risk of cerebrovascular diseases. However, the association between noise exposure and cerebrovascular diseases has not received much attention. To our knowledge, this is the first study to examine prospectively the association between noise exposure and cerebrovascular diseases.

\section{Methods}

The details of the Japan Collaborative Cohort (JACC) Study, which was sponsored by the Ministry of Education, Culture, Sports, Science, and Technology of Japan, have been described previously ${ }^{12,18,19)}$. Briefly, this prospective cohort study involved a total of 110,792 subjects $(46,465$ male and 64,327 female) who were aged between 40 and $79 \mathrm{yr}$ at the time of recruitment in 45 areas of Japan between 1988 and 1990. In 22 out of the 45 areas, all residents living in a given target area were regarded as study subjects. In 20 areas, those who had undertaken a basic health examination conducted under the Health and Medical Service Law for the Aged were invited to participate in the study. In two areas, the study subjects consisted of health examinees plus volunteers. In one area, subjects were defined based on the health check-up for atomic bomb survivors. The response rates were obtainable from 17 of 22 areas which included all living residents as the subjects: the average response rate was $83 \%{ }^{19)}$.

The follow-up survey was conducted using population registries in local municipalities to determine the vital and residential status of the cohort in each area. All subjects who moved out of the study areas were treated as censored subjects. All deaths that occurred in the cohort were ascertained by death certificated from local public health centers in the study areas with permission from the Ministry of Public Management, Home Affairs, Post and Telecommunications, Japan. For deceased subjects, the causes of death were recorded from the death certificates held at the regional health centres and were classified according to the International Classification of Disease 10th Revision (ICD-10) as follows: cerebrovascular diseases (I60 to I69), subarachnoid haemorrhage (I60.0-I60.9), intracerebral haemorrhage (I61.0-I61.9), and cerebral infarction(I63.0-I63.9).

The present analysis included follow-up data that were collected until the end of 2003. This study was approved by the Ethics Committees of Nagoya University and the University of Tsukuba, Japan.

\section{Baseline survey and data retrieval for analysis}

In order to isolate the appropriate data for our analysis, the study group was initially restricted to male workers whose baseline ages ranged from 40 to 59 years $(n=20,630)$ since the most common retiring age is 60 in Japan. Of these workers, 14,651 provided information on the perceived nose level in their workplace. We then further limited this group to individuals free $(n=14,568)$ of cerebrovascular diseases $(n=83)$. Of the 1,064 deaths that occurred during the 190,777 person-years of followup, 98 deaths were attributed to cerebrovascular diseases, 27 deaths were attributed to subarachnoid haemorrhage, 35 deaths were attributed to intracerebral haemorrhage, and 25 deaths were attributed to cerebral infarction.

All subjects completed a self-administered questionnaire at the baseline. This included the following question regarding perceived noise exposure at work which they have mostly experienced during their working life: "During your working life, until the present, do you feel extreme noise at the workplace?" The selfadministered questionnaire also inquired about other baseline characteristics that could potentially be related to mortality. These included the following: smoking status (never, former or current smoker); alcohol intake (non-habitual drinker, former habitual drinker or habitual drinkers of ethanol at 1 to 22,23 to 44 , and $>44 \mathrm{~g}$ per day), past medical history (hypertension or diabetes); educational level (school attendance beyond 18th birthday, until the age of 16 to $17 \mathrm{yr}$ or until the age of 15 yr or less); degree of perceived mental stress in daily life (frequent, occasional, rarely or never); hours of walking $(<0.5,0.5,0.6$ to 0.9 , and $1.0 \leq$ per day), hours of exercise ( $<1,1$ to 2,3 to 4 , and $5 \leq$ per week), type of job (office worker, manual worker or other), and patterns of shift work (mainly daytime, fixed-night shift, or rotating-shift work).

\section{Statistical analysis}

The Cox proportional-hazards model was used to estimate the hazard ratios (HRs) of perceived noise exposure at work for each cause of death: cerebrovascular diseases, subarachnoid haemorrhage, intracerebral haemorrhage, and cerebral infarction ${ }^{20)}$. The model included age divided into 5-yr groups and body mass index (BMI) divided into quartiles, and the potential confounding factors listed above. The assumptions of the proportional-hazards model were checked by including the interaction terms of the predictors and a function of survival time ${ }^{21)}$. All calculations were performed using the SAS statistical software package ${ }^{22)}$.

\section{Results}

Of the 14,568 men, 5,405 $(37.1 \%)$ reported that they were exposed to noise at work (Table 1). Compared to those who did not perceive noise exposure at work, men who had perceived noise exposure at work reported a higher prevalence of myocardial infarction (0.9 vs $1.4 \%)$, a higher prevalence of current smoking ( 54.5 vs $58.7 \%$ ), and higher perceived levels of frequent and occasional stress (26.6 vs $35.5 \%$ ). In addition, those who had perceived noise exposure at work were more likely to 
Table 1. Selected baseline characteristics according to perceived noise exposure at work

\begin{tabular}{|c|c|c|c|}
\hline & \multicolumn{3}{|c|}{ Perceived noise exposure at work } \\
\hline & No & Yes & $p^{*}$ \\
\hline $\mathrm{n}$ & 9,163 & 5,405 & \\
\hline Mean age (SD) & $49.3(5.9)$ & $49.3(5.9)$ & 0.928 \\
\hline Mean body mass index (SD) & $23.1(2.7)$ & $22.9(2.7)$ & $<0.001$ \\
\hline Past history of hypertension (\%) & 12.5 & 13.1 & 0.341 \\
\hline Past history of myocardial infarction (\%) & 0.9 & 1.4 & 0.005 \\
\hline Past history of diabetes (\%) & 4.7 & 4.2 & 0.168 \\
\hline Smoking $(\%)$ & & & $<0.001$ \\
\hline Never smoker & 23.2 & 18.8 & \\
\hline Current smoker & 54.5 & 58.7 & \\
\hline Former smoker & 19.5 & 20.1 & \\
\hline Alcohol Drinking $(\%)$ & & & $<0.001$ \\
\hline Non-habitual drinker & 15.4 & 14.9 & \\
\hline Former drinker & 2.7 & 3.2 & \\
\hline Habitual drinker & 79.2 & 78.7 & \\
\hline Ethanol at 1 to $22 \mathrm{~g}$ per day & 34.8 & 30.8 & \\
\hline Ethanol at 23 to $44 \mathrm{~g}$ per day & 26.0 & 26.9 & \\
\hline Ethanol at $>44$ g per day & 11.8 & 14.5 & \\
\hline Hours of walking per day & & & $<0.001$ \\
\hline $1.0 \leq$ & 44.8 & 50.0 & \\
\hline $0.6-0.9$ & 20.0 & 18.0 & \\
\hline 0.5 & 20.1 & 15.9 & \\
\hline$<0.5$ & 12.8 & 13.8 & \\
\hline Hours of exercise per week & & & $<0.001$ \\
\hline $5 \leq$ & 4.6 & 4.0 & \\
\hline $3-4$ & 6.7 & 4.9 & \\
\hline $1-2$ & 19.2 & 17.2 & \\
\hline$<1$ & 67.8 & 71.9 & \\
\hline Perceived mental stress (\%) & & & $<0.001$ \\
\hline Frequent & 11.9 & 18.0 & \\
\hline Occasional & 14.7 & 17.5 & \\
\hline Rarely & 54.2 & 48.9 & \\
\hline Never & 12.0 & 8.4 & \\
\hline Educational Levels (\%) & & & $<0.001$ \\
\hline$<15 \mathrm{yr}$ & 22.9 & 33.3 & \\
\hline $15-17 \mathrm{yr}$ & 13.1 & 17.0 & \\
\hline $18 \mathrm{yr} \leq$ & 59.5 & 44.2 & \\
\hline Engaging in shift work & 9.3 & 20.9 & $<0.001$ \\
\hline Job Type $(\%)$ & & & $<0.001$ \\
\hline Office work & 26.7 & 11.1 & \\
\hline Manual work & 46.6 & 67.1 & \\
\hline Others & 24.1 & 19.4 & \\
\hline
\end{tabular}

* $p$ values were derived from $t$-test or chi-square test.

have been engaged in shift work and manual work, and have left school earlier.

No significant difference in the risk of death due to cerebrovascular diseases, death due to subarachnoid haemorrhage, or death due to cerebral infarction was observed between those who perceived noise exposure at work and those who did not (Table 2). However, perceived noise exposure at work was associated with a 2-fold increase in the risk of intracerebral haemorrhage $(\mathrm{HR}=2.38,95 \%$ Confidence interval (CI): 1.20, 4.71, $p=0.013$ ). Adjustment for multiple potential risk factors did not significantly alter the results. In the multivariable model for intracerebral haemorrhage, the HR of hypertension was 2.80 (95\%CI: $1.28,6.11, p=0.001)$, that 
Table 2. Hazard ratios of perceived noise exposure at work associated with cause-specific mortality

\begin{tabular}{|c|c|c|c|c|c|c|c|c|}
\hline \multirow[t]{2}{*}{ Cause of death } & \multirow{2}{*}{$\begin{array}{l}\text { Perceived noise } \\
\text { exposure at work }\end{array}$} & \multirow[b]{2}{*}{ Deaths * } & \multicolumn{3}{|c|}{ Age-adjusted } & \multicolumn{3}{|c|}{ Multivariable $^{\dagger}$} \\
\hline & & & HR & $95 \% \mathrm{CI}$ & $p$ & HR & $95 \% \mathrm{CI}$ & $p$ \\
\hline \multirow[t]{2}{*}{ Cerebrovascular diseases } & no & 51 & reference & & & reference & & \\
\hline & yes & 47 & 1.47 & $0.99,2.19$ & 0.058 & 1.31 & $0.85,2.02$ & 0.219 \\
\hline \multirow[t]{2}{*}{ Intracerebral haemorrhage } & no & 14 & reference & & & reference & & \\
\hline & yes & 21 & 2.38 & $1.20,4.71$ & 0.013 & 2.11 & $1.01,4.40$ & 0.047 \\
\hline \multirow[t]{2}{*}{ Cerebral infarction } & no & 12 & reference & & & reference & & \\
\hline & yes & 13 & 1.66 & $0.75,3.65$ & 0.210 & 1.74 & $0.73,4.10$ & 0.209 \\
\hline \multirow[t]{2}{*}{ Subarachnoid haemorrhage } & no & 18 & reference & & & reference & & \\
\hline & yes & 9 & 0.78 & $0.35,1.75$ & 0.552 & 0.67 & $0.28,1.60$ & 0.367 \\
\hline
\end{tabular}

*Person-years are 70,466 for the subjects with perceived noise exposure at work, and 120,311 for those who without it. $\dagger$ Multivariable model included age, smoking, alcohol consumption, educational level, perceived mental stress, past medical history, body mass index, hours of walking, hours of exercise, shift work, and job type. HR: Hazard ratio, CI: Confidence interval.

Table 3. Multivariate hazard ratios (HRs) of noise exposure for intracerebral haemorrhage in separate strata of hypertension

\begin{tabular}{|c|c|c|c|c|c|c|c|}
\hline \multirow[b]{3}{*}{ Strata } & \multicolumn{7}{|c|}{ Perceived noise exposure at work } \\
\hline & \multicolumn{2}{|c|}{ No } & \multicolumn{5}{|c|}{ Yes } \\
\hline & Person-year & Deaths $^{\dagger}$ & Person-year & Deaths $^{\dagger}$ & $\mathrm{HR}^{*}$ & $95 \% \mathrm{CI}$ & $p$ \\
\hline \multicolumn{8}{|c|}{ Hypertension } \\
\hline No & 105,680 & 12 & 61,551 & 12 & 1.21 & $0.51,2.86$ & 0.673 \\
\hline Yes & 14,631 & 2 & 8,915 & 9 & 55.6 & $3.61,857$ & 0.004 \\
\hline
\end{tabular}

* Multivariable model included age, smoking, alcohol consumption, educational level, perceived mental stress, past medical history, body mass index, hours of walking, hours of exercise, shift work, and job type. †Number of deaths from intracerebral haemorrhage. HR: Hazard ratio, CI: Confidence interval.

of less education (school attendance until the age of 17 yr) was $2.63(95 \% \mathrm{CI}: 1.12,6.16, p=0.026)$, and that of walking hours of $\geq 1.0$ per day was 0.35 (95\% CI: 0.14 , $0.87, p=0.023)$. When the Cox proportional-hazard model included the interaction term multiplying hypertension and perceived noise exposure at work in order to assess the modifying effects of hypertension, the HR of perceived noise exposure at work was $1.42(95 \% \mathrm{CI}=0.61$, $3.28, p=0.415)$, that of hypertension was $0.96(95 \%$ $\mathrm{CI}=0.20,4.53, p=0.956)$, and that of the interaction term was $4.95(95 \% \mathrm{CI}=0.84,29.24, p=0.077)$.

Furthermore, multivariable HRs of perceived noise exposure at work for intracerebral haemorrhage were estimated for separate strata of hypertension (Table 3). Compared with those who did not perceive noise exposure at work, the HRs of those who perceived noise exposure at work for intracerebral haemorrhage were as follows: $55.6(95 \% \mathrm{CI}=3.61,857, p=0.004)$ among subjects with hypertension; 1.21 (95\% CI=0.51, 2.86, $p=0.673)$ among subjects without hypertension.

\section{Discussion}

The present study shows that perceived noise exposure at work increased the risk of death due to intracerebral hemorrhage, but did not increase the risk of death due to subarachnoid hemorrhage or cerebral infarction among Japanese male workers. When analyses were stratified by hypertension, the association of perceived noise exposure at work with the risk of intracerebral hemorrhage was only observed among the subjects with hypertension.

Noise exposure can cause several health effects, resulting from biochemical, physiological, and psychosocial changes $^{6}$. Although there has been no evidence linking noise exposure with cerebrovascular diseases, some suggestions have been given in previous studies that have examined the association of noise exposure with coronary heart disease and hypertension ${ }^{1-6)}$. It is supposed that noise exerts its health effects via stress, since noise often raises stress in various ways ${ }^{6}$. Stress increases blood pressure levels and heart rates resulting from activation of the sympathetic nervous systems ${ }^{7}$. Hypertension and tachycardia increase the risk of cerebrovascular diseases and coronary heart disease ${ }^{23)}$. This is backed by the stronger relation of perceived noise exposure at work with intracerebral hemorrhage, but not with cerebral infarction 
or subarachnoid hemorrhage, found in the present study, since hypertension is a stronger risk factor for intracerebral hemorrhage ${ }^{24)}$. In the present study, subjects who perceived noise exposure at work actually reported higher perceived levels of frequent and occasional stress than those who did not. However, adjustment for perceived mental stress and history of hypertension did not substantially change the results, which suggests other mechanisms.

Notably, perceived noise exposure at work dramatically increased the risk of death due to intracerebral hemorrhage among the subjects with hypertension, although the relatively small number of cases gave a wide range for the $95 \%$ confidential interval. This may suggest that subjects with hypertension are highly susceptible to noise exposure perhaps due to instability of blood pressure when exposed to exogenous stimuli, including noise exposure. Various studies have suggested that subjects with hypertension have exaggerated blood pressure reactivity to stress compared with normotensives ${ }^{25-29)}$. In addition, a study reported that the excess mortality from coronary heart disease and stroke associated with mental stress tended to be more evident among those with hypertension who may have more advanced atherosclerosis compared with those with normal blood pressure $^{12)}$. It has also been suggested that mental stress may trigger the clinical events of coronary heart disease and cerebrovascular diseases when atherosclerosis is advanced $^{11,12,30)}$.

Another plausible explanation for the association of perceived noise exposure at work with the risk of death due to intracerebral hemorrhage is the fact that occupational noise exposure is one of the proxies of socioeconomic status, which has been recognized as main determinant of individuals' health ${ }^{31,32)}$. However, the multivariable model still showed a significant relationship of noise with intracerebral hemorrhage even after adjustment for educational level, type of job, and shift work, which are often considered as major proxies of socioeconomic status as well. Therefore, the association of perceived noise at work with intracerebral hemorrhage cannot be solely explained by socioeconomic status in relation to noise exposure at work.

Some limitations should be mentioned. First, we used perceived measurement of noise exposure at work rather than sound level measurement or personal dosimeter. In terms of mental stress, however, we assume that perceived noise exposure is more useful than sound level measurement or personal dosimeter, since appraisal of noise exposure is mediated by personal characteristics such as attitude and coping style ${ }^{33)}$. However, discussion of a dose-response relationship of perceived noise exposure with the risk of cerebrovascular diseases is ineligible in the present study. The second limitation is the residual confounding of the association between perceived noise exposure at work and risk of cerebrovascular diseases. Although we adjusted for the selected factors that potentially relating to mortality, the possible influence of other risk factors, lifestyles, and psychosocial factors might still remain. Third, we used the mortality data as end points rather than incidence data, which may lead to misclassification in the diagnosis of stroke subtypes. However, the widespread use of computer tomography in local hospitals since the 1980 s has probably made the diagnosis of stroke subtypes reported on death certificates sufficiently accurate $^{34,35)}$. In addition, given mental stress showed stronger relationships with fatal stroke than non-fatal stroke ${ }^{11,13,15)}$, the use of mortality data may be legitimate to support our hypothesis. Finally, this study did not examine the noise effect relating to specific type of job or noise, such as traffic, mechanical, or construction noise, although this study did examine a broad range of workers retrieved from the cohorts regarded as representing the chosen areas.

The present study provides epidemiologic evidence that perceived noise exposure at work potentially increases the risk of death due to intracerebral hemorrhage among subjects with hypertension, but not among subjects without hypertension, which may draw attention for occupational health policy. Although the underlying mechanisms are uncertain, hypertensive individuals with perceived noise exposure at work should be regarded as a high-risk group for intracerebral hemorrhage.

Acknowledgments: The authors sincerely express their appreciation to Dr. Kunio Aoki, Professor Emeritus, Nagoya University School of Medicine and the former chairman of the Ministry of Education, Science, Sports and Culture of Japan (Monbusho) ECC, and Dr. Haruo Sugano, the former Director, of the Cancer Institute, Tokyo, who greatly contributed to the initiation of the JACC Study.

The JACC Study has been supported by Grants-in-Aid for Scientific Research from the Ministry of Education, Science, Sports and Culture of Japan (Nos. 61010076, 62010074, 63010074, 1010068, 2151065, 3151064, 4151063, 5151069, 6279102, and 11181101).

\section{The member list of the JACC Study Group}

The present investigators involved, with the coauthorship of this paper, in the JACC Study and their affiliations are as follows: Dr. Akiko Tamakoshi (present chairman of the study group), Nagoya University Graduate School of Medicine; Dr. Mitsuru Mori, Sapporo Medical University School of Medicine; Dr. Yutaka Motohashi, Akita University School of Medicine; Dr. Ichiro Tsuji, Tohoku University Graduate School of Medicine; Dr. Yoshikazu Nakamura, Jichi Medical School; Dr. Hiroyasu Iso, Institute of Community Medicine, University of Tsukuba; Dr. Haruo Mikami, 
Chiba Cancer Center; Dr. Yutaka Inaba, Juntendo University School of Medicine; Dr. Yoshiharu Hoshiyama, University of Human Arts and Sciences; Dr. Hiroshi Suzuki, Niigata University School of Medicine; Dr. Hiroyuki Shimizu, Gifu University School of Medicine; Dr. Hideaki Toyoshima, Nagoya University Graduate School of Medicine; Dr. Kenji Wakai, Aichi Cancer Center Research Institute; Dr. Shinkan Tokudome, Nagoya City University Graduate School of Medical Sciences; Dr. Yoshinori Ito, Fujita Health University School of Health Sciences; Dr. Shuji Hashimoto, Fujita Health University School of Medicine; Dr. Shogo Kikuchi, Aichi Medical University School of Medicine; Dr. Akio Koizumi, Graduate School of Medicine and Faculty of Medicine, Kyoto University; Dr. Takashi Kawamura, Kyoto University Center for Student Health; Dr. Yoshiyuki Watanabe, Kyoto Prefectural University of Medicine Graduate School of Medical Science; Dr. Tsuneharu Miki, Graduate School of Medical Science, Kyoto Prefectural University of Medicine; Dr. Chigusa Date, Faculty of Human Environmental Sciences, Mukogawa Women's University; Dr. Kiyomi Sakata, Wakayama Medical University; Dr. Takayuki Nose, Tottori University Faculty of Medicine; Dr. Norihiko Hayakawa, Research Institute for Radiation Biology and Medicine, Hiroshima University; Dr. Takesumi Yoshimura, Fukuoka Institute of Health and Environmental Sciences; Dr. Akira Shibata, Kurume University School of Medicine; Dr. Naoyuki Okamoto, Kanagawa Cancer Center; Dr. Hideo Shio, Moriyama Municipal Hospital; Dr. Yoshiyuki Ohno, Asahi Rosai Hospital; Dr. Tomoyuki Kitagawa, Cancer Institute of the Japanese Foundation for Cancer Research; Dr. Toshio Kuroki, Gifu University; and Dr. Kazuo Tajima, Aichi Cancer Center Research Institute.

\section{References}

1) Morrell S, Taylor R and Lyle D: A review of health effects of aircraft noise. Aust N Z J Public Health 21, 221-236 (1997)

2) Babisch W, Ising H, Elwood PC, Sharp DS and Bainton D: Traffic noise and cardiovascular risk: the Caerphilly and Speedwell studies, second phase. Risk estimation, prevalence, and incidence of ischemic heart disease. Arch Environ Health 48, 406-413 (1993)

3) Babisch W, Ising $H$ and Gallacher JE: Health status as a potential effect modifier of the relation between noise annoyance and incidence of ischaemic heart disease. Occup Environ Med 60, 739-745 (2003)

4) Babisch W, Ising H, Gallacher JE, Sweetnam PM and Elwood PC: Traffic noise and cardiovascular risk: the Caerphilly and Speedwell studies, third phase-10-year follow up. Arch Environ Health 54, 210-216 (1999)

5) Davies HW, Teschke K, Kennedy SM, Hodgson MR, Hertzman C and Demers PA: Occupational exposure to noise and mortality from acute myocardial infarction. Epidemiology 16, 25-32 (2005)
6) van Kempen EE, Kruize H, Boshuizen HC, Ameling $\mathrm{CB}$, Staatsen BA and de Hollander AE: The association between noise exposure and blood pressure and ischemic heart disease: a meta-analysis. Environ Health Perspect 110, 307-317 (2002)

7) Kelsey RM, Blascovich J, Tomaka J, Leitten CL, Schneider TR and Wiens S: Cardiovascular reactivity and adaptation to recurrent psychological stress: effects of prior task exposure. Psychophysiology 36, 818-831 (1999)

8) Moberg E, Kollind M, Lins PE and Adamson U: Acute mental stress impairs insulin sensitivity in IDDM patients. Diabetologia 37, 247-251 (1994)

9) Grignani G, Pacchiarini L, Zucchella M, Tacconi F, Canevari A, Soffiantino F and Tavazzi L: Effect of mental stress on platelet function in normal subjects and in patients with coronary artery disease. Haemostasis 22, 138-146 (1992)

10) Ghiadoni L, Donald AE, Cropley M, Mullen MJ, Oakley G, Taylor M, O'Connor G, Betteridge J, Klein N, Steptoe A and Deanfield JE: Mental stress induces transient endothelial dysfunction in humans. Circulation 102, 2473-2478 (2000)

11) Truelsen $T$, Nielsen N, Boysen G and Gronbaek M: Self-reported stress and risk of stroke: the Copenhagen City Heart Study. Stroke 34, 856-862 (2003)

12) Iso $H$, Date $C$, Yamamoto $A$, Toyoshima $H$, Tanabe $N$, Kikuchi S, Kondo T, Watanabe Y, Wada Y, Ishibashi T, Suzuki H, Koizumi A, Inaba Y, Tamakoshi A and Ohno Y: Perceived mental stress and mortality from cardiovascular disease among Japanese men and women: the Japan Collaborative Cohort Study for Evaluation of Cancer Risk Sponsored by Monbusho (JACC Study). Circulation 106, 1229-1236 (2002)

13) Ohlin B, Nilsson PM, Nilsson JA and Berglund G: Chronic psychosocial stress predicts long-term cardiovascular morbidity and mortality in middle-aged men. Eur Heart J 25, 867-873 (2004)

14) Franke WD, Ramey SL and Shelley MC 2nd: Relationship between cardiovascular disease morbidity, risk factors, and stress in a law enforcement cohort. J Occup Environ Med 44, 1182-1189 (2002)

15) May M, McCarron P, Stansfeld S, Ben-Shlomo Y, Gallacher J, Yarnell J, Davey Smith G, Elwood P and Ebrahim S: Does psychological distress predict the risk of ischemic stroke and transient ischemic attack? The Caerphilly Study. Stroke 33, 7-12 (2002)

16) Harmsen P, Rosengren A, Tsipogianni A and Wilhelmsen L: Risk factors for stroke in middle-aged men in Goteborg, Sweden. Stroke 21, 223-229 (1990)

17) Greenwood DC, Muir KR, Packham CJ and Madeley RJ: Coronary heart disease: a review of the role of psychosocial stress and social support. J Public Health Med 18, 221-231 (1996)

18) Ohno Y and Tamakoshi A: Japan collaborative cohort study for evaluation of cancer risk sponsored by monbusho (JACC study). J Epidemiol 11, 144-150 (2001)

19) Tamakoshi A, Toshimura T, Inaba $Y$, Ito $Y$, Watanabe Y, Fukuda K and Iso H: Profile of JACC Study. J 
Epidemiol 15, S4-S8 (2005)

20) Cox DR, Oakes D. Analysis of Survival Data. New York: Chapman and Hall, 1984.

21) Hosmer JD, Lemeshow S. Applied Survival Analysis: Regression Modeling of Time to Event Data. New York: John Wiley \& Sons Inc., 1999.

22) SAS Institute Inc. SAS/STAT User's Guide, Release 6.03. Cary, North Carolina: SAS Institute Inc., 1988.

23) Palatini $P$ and Julius $S$ : Heart rate and the cardiovascular risk. J Hypertens 15, 3-17 (1997)

24) Shimamoto T, Komachi Y, Inada H, Doi M, Iso H, Sato S, Kitamura A, Iida M, Konishi M, Nakanishi N, Terao A, Naito Y and Kojima S: Trends for coronary heart disease and stroke and their risk factors in Japan. Circulation 79, 503-515 (1989)

25) Fredrikson $M$ and Matthews KA: Cardiovascular responses to behavioral stress and hypertension: a metaanalytic review. Ann Behav Med 12, 30-39 (1990)

26) Fredrikson M, Blumenthal JA, Evans DD, Sherwood A and Light $\mathrm{KC}$ : Cardiovascular responses in the laboratory and in the natural environment: is blood pressure reactivity to laboratory-induced mental stress related to ambulatory blood pressure during everyday life? J Psychosom Res 33, 753-762 (1989)

27) Fredrikson M, Dimberg U, Frisk-Holmberg $M$ and Strom G: Haemodynamic and electrodermal correlates of psychogenic stimuli in hypertensive and normotensive subjects. Biol Psychol 15, 63-73 (1982)

28) Tsai PS, Yucha CB, Nichols WW and Yarandi H:
Hemodynamics and arterial properties in response to mental stress in individuals with mild hypertension. Psychosom Med 65, 613-619 (2003)

29) Tuomisto MT: Intra-arterial blood pressure and heart rate reactivity to behavioral stress in normotensive, borderline, and mild hypertensive men. Health Psychol 16, 554-565 (1997)

30) Gullette EC, Blumenthal JA, Babyak M, Jiang W, Waugh RA, Frid DJ, O'Connor CM, Morris JJ and Krantz DS: Effects of mental stress on myocardial ischemia during daily life. JAMA 277, 1521-1526 (1997)

31) Berkman L, Kawachi I. Social Epidemiology. New York: Oxford University Press, 2000.

32) Marmot M, Wilkinson R. Social Determinants of Health. New York: Oxford University Press, 1999.

33) Health Council of the Netherlands. Public health impact of large airports. Report No.1999/14E. The Hague: Health Council of the Netherlands, 1999.

34) Iso H, Jacobs DR Jr and Goldman L: Accuracy of death certificate diagnosis of intracranial hemorrhage and nonhemorrhagic stroke. The Minnesota Heart Survey. Am J Epidemiol 132, 993-998 (1990)

35) Sankai T, Miyagaki T, Iso H, Shimamoto T, Iida M, Tanigaki M, Naito Y, Sato S, Kiyama M and Kitamura A: A population-based study of the proportion by type of stroke determined by computed tomography scan. Nippon Koshu Eisei Zasshi 38, 901-909 (1991) (in Japanese) 